\title{
A Model to Getting the Best Temperature of Bathtub
}

\author{
Kai Wang ${ }^{1, a}$, \\ ${ }^{1}$ North China Electric Power University, Baoding 071003, China \\ awk1248052647@126.com
}

Keywords: Bathtub, Thermodynamics, Best temperature.

\begin{abstract}
In this paper, we use the first law of thermodynamics to obtain a differential equation. After comprehensively consider about evaporative heat loss, convection heat loss and radiation heat loss, we develop the model to discuss the temperature of the bathtub water for different time and different flow rate. The best velocity of hot water at inlet and the best temperature of hot water at inlet can be obtained based on this model so that we can determine the best strategy the person in the bathtub can adopt to keep the temperature even throughout the bathtub. Afterwards, we analyze the effect of some critical factors such as the shape/volume of bathtub, the shape/volume/temperature of body, motions and bubble bath additive to water temperature. And also, we discuss other factors that we consider important, for instance, the installation location of faucet and over flow hole.
\end{abstract}

\section{Introduction}

When we finish work to take a bath, Water temperature comfort for us is very much. Of course, the temperature of the water will not stand still, The temperature of the water will be under the influence of external factors rapidly reduced. As opposed to that most people use the regular bathtub without a secondary heating system because of low price. Therefore, ${ }^{[1]}$ we through a mathematical model to find the best way to keep one's bath temperature.

Based on the analysis, we figure out that the reason for the decrease in water temperature is due to the heat loss in the system such as evaporative heat loss, convection heat loss and radiation heat loss, heat loss by the contact between water and the wall of the bathtub. In addition, people will absorb the heat from the water. Based on overall consideration of various factors, we develop a model.

\section{Model Developing}

\subsection{Introduction}

A heat transfer model was developed to discuss the temperature of the bathtub water for different time and different flow rate by using the first law of thermodynamics. Based on the data of relative documents and our previous work, the optimum temperature for adding water was computed, and then the appropriate flow rate was decided. ${ }^{[2]}$

\subsection{The Foundation of Model}

When we try to obtain the temperature of the bathtub water in space and time, we use the Fourier 3-D differential equation of heat transfer model. Fourier 3-D differential equation of heat transfer is a method of obtaining solution of temperature field in space. So we can use it to solve the temperature field distribution of the water in the bathtub because this temperature field is satisfied with the condition of the model. And we add the appropriate boundary conditions and heat source heating methods during solving the model.

Partial differential equation of the temperature distribution. We use the Fourier partial differential equation to establish the following equation with the parameters of the bathtub, then, we find the temperature field mathematical model in time and space:

$$
\frac{\partial \mathrm{T}}{\partial \mathrm{t}}=\frac{\lambda}{C \rho}\left(\frac{\partial^{2} T}{\partial x^{2}}+\frac{\partial^{2} T}{\partial y^{2}}+\frac{\partial^{2} T}{\partial z^{2}}\right)
$$


Calculate the distance between the goal point and the original point. If the location of the temperature field is different, the corresponding temperature will be different, but it will be in accordance with the above partial differential equation according to a certain law of distribution.

$$
\mathrm{r}=\sqrt{x^{2}+y^{2}+z^{2}}
$$

We integrate above formulas, and obtain the following formula. Then we collect the statistics to solve the model.

$$
\mathrm{T}(\mathrm{r}, \mathrm{t})-T_{0}=\frac{2 q \Delta t}{c \rho(4 \pi a t)^{\frac{3}{2}}} e^{-\frac{r^{2}}{4 a t}}
$$

The water temperature of the bathtub in space is shown in the Fig.1. This figure is based on the mathematical model solution, the red part represents the place where the temperature is relatively high in the temperature field. Around the blue place where the temperature is relatively low, the heat source is on the top of the red, heat source transfer heat to the whole temperature field, heat is transferring in the direction of decreasing temperature field.

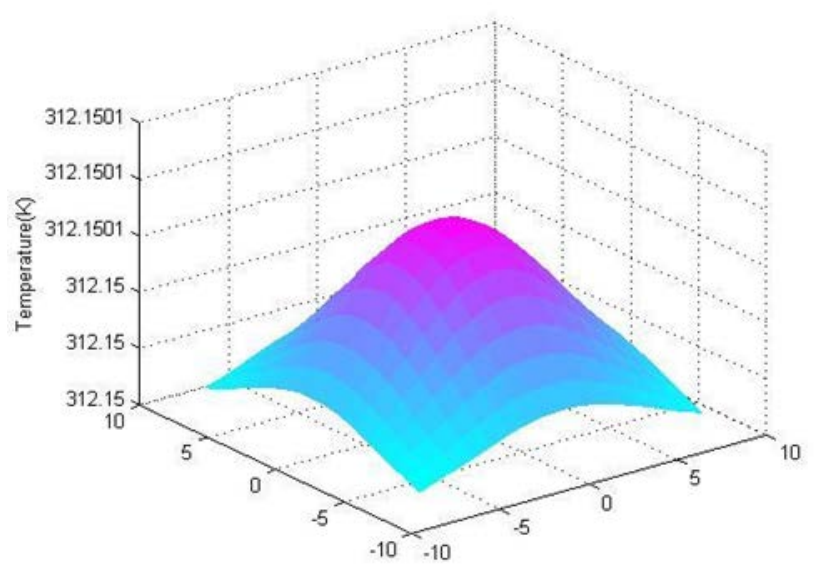

Fig.1 The temperature of bathtub water in space

By the above model, it is easy to see that the change of temperature distribution in the space position. Then we establish a thermodynamics model to solve the problem of heat loss.

$$
Q_{1}=\lambda_{1} A_{1} \frac{T-T_{2}}{\delta_{1}}
$$

$\mathrm{Q}_{1}$ is the heat loss through the wall heat transfer, it with the bathtub and bathroom wall contact area and its shape and area.

We can know the bathroom temperature is the function of ' $t$ ' by the knowledge of non-steady heat transfer

$$
T_{2}=299.2+298.15 e^{-t}
$$

$\mathrm{Q}_{2}$ is the faucet provide heat for bath.The water in the bathtub after cooling, hot water through the tap into the bath crock is used to raise the temperature.

$$
Q_{2}=c \rho A_{2} \frac{b}{T_{2}}\left(T_{1}-T\right)
$$

$\mathrm{Q}_{3}$ represents the endothermic formula:

$$
Q_{3}=\lambda_{3}(0.006 h+0.0128 w-0.159) \frac{T-T_{3}}{\delta_{3}}
$$

Finally we can get the following equation:

$$
\mathrm{c} \rho \mathrm{v} \Delta \mathrm{t}+Q_{1} \Delta t+Q_{3} \Delta t+Q_{4} \Delta t=\mathrm{Q}_{2} \Delta \mathrm{t}
$$

$\mathrm{Q}_{3}$ is a empirical formula. ${ }^{[3]}$ 


\subsection{Solution and Result}

We can get optimization results by the model above, But we have to use a speed stream function to get the results. Therefore, we need a process of constantly adjust speed function, only by constantly optimize speed function, to obtain the optimal solution.

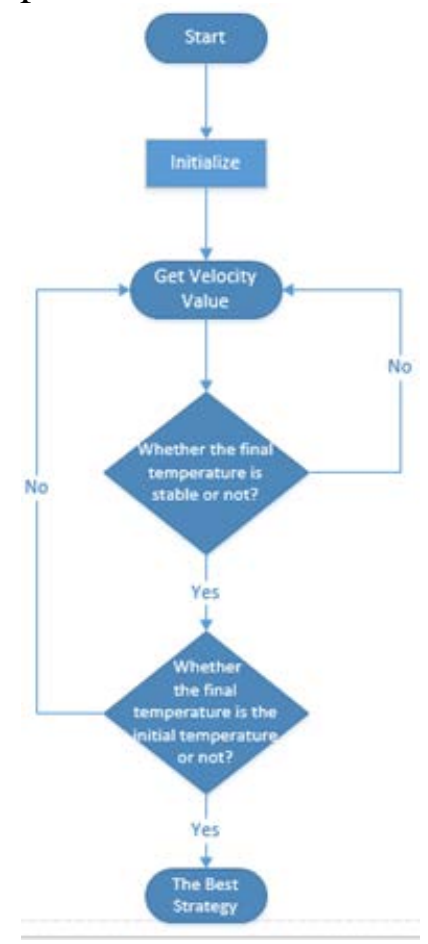

Fig. 2 Process to obtain the optimum strategy

We need to initialize the data before finding the optimal flow rate, simplify the model. So finding the best flow rate will become more convenient. Through the thermodynamic model, we obtain Fig.2 which described the bathroom temperature change with the time.

\section{References}

[1] Asdrubali F. A scale model to evaluate water evaporation from indoor swimming pools[J]. Energy and buildings, 2009, 41(3): 311-319.

[2] Maximum heating temperature of water heater. http : // baike. baidu. com/ link ?url =U jcB NREk Hj8 xfE h EH- XsXwi 9RXcMkJ U0ZPk z_q_nDr4 j n B -F7 d2A m8Uv_S IX X f XRcD dhHvQO DlhE-o2E C7jejK\#1_1

[3] The most suitable temperature of bath http: //szb. Dlxww .com /xsb / html/ 2010- 05 /10 /content _350609.htm 